\title{
Gerenciamento de resultados e ciclo eleitoral em municípios brasileiros
}

Fábio Moraes da Costa 1

Fernando Henrique Ferreira Carneiro Leão ${ }^{1}$

1 FUCAPE Business School, Vitória - ES, Brasil

O regime de competência tem sido gradualmente implementado no setor público brasileiro, ampliando o uso de estimativas para sua aplicação. Apesar de ter o objetivo de contribuir para a tomada de decisão, sabe-se que julgamentos também podem ser empregados para gerenciar a percepção sobre a performance. Assim, este estudo tem como objetivo avaliar a relação entre o ciclo eleitoral e a prática de gerenciamento de resultados nos municípios brasileiros. Os testes de análise da distribuição de frequência indicam que há descontinuidade em torno do resultado nulo, além de uma relação positiva entre o nível de gerenciamento de resultados e a probabilidade de o município apresentar um superávit logo acima de zero ("pequeno superávit"). Também foi evidenciado o aumento dos níveis de gerenciamento em períodos pré-eleitorais, bem como ambientes de maior nível de competição política. Portanto, há evidências de práticas de gerenciamento de resultados em função do ciclo eleitoral. $O$ trabalho pretende suprir um pouco da falta de informação sobre o tema no setor público, bem como da identificação de padrões de comportamento oportunista por parte dos gestores locais brasileiros e sua inter-relação com os ciclos eleitorais.

Palavras-chave: gerenciamento de resultados; ciclo eleitoral; municípios.

\section{Gestión de ganancias y ciclo electoral en municipios brasileños}

El régimen de competencia se está implementando gradualmente en el sector público brasileño, lo que resulta en el uso de estimaciones para su aplicación. Aunque el objetivo principal es contribuir a la toma de decisiones, los juicios también se pueden utilizar para gestionar las percepciones sobre el rendimiento. Por lo tanto, el propósito de este estudio es evaluar la relación entre el ciclo electoral y las prácticas de gestión de ingresos en los municipios brasileños. Las pruebas basadas en la distribución de frecuencias indican que hay una discontinuidad alrededor de cero ganancias y que los municipios con “pequeños excedentes” presentan niveles más altos de los accruals discrecionales. Finalmente, presentamos evidencia empírica de que hay un aumento en el nivel de gestión de ingresos en los períodos preelectorales, que se mitiga en entornos con mayor competencia política. En conjunto, la evidencia empírica indica que existe una relación entre las prácticas de gestión de ingresos y el ciclo electoral. El estudio contribuye a la literatura cada vez mayor sobre la calidad de los ingresos en el sector público, ayudando a identificar circunstancias que pueden conducir a un comportamiento oportunista por parte de los administradores públicos, especialmente en relación con las elecciones.

Palabras clave: gestión de ingresos; ciclo electoral; municipios.

\section{Earnings management and electoral cycle in Brazilian municipalities}

The accruals basis of accounting is gradually being implemented in the Brazilian public sector, which has increased the use of estimates. Although the judgments' main objective is to contribute to decision-making, they can also be used to manage perceptions on performance. This study aims to evaluate the relationship between the electoral cycle and earnings management practices in Brazilian municipalities. Tests based on frequency distribution were conducted, indicating a discontinuity around zero earnings and suggesting that municipalities with 'small surpluses' present higher levels of discretionary accruals. Finally, we gathered empirical evidence of an increase in the level of earnings management in the pre-electoral periods, which is mitigated in environments with strong political competition. The empirical findings indicate a correlation between earnings management practices and the electoral cycle. The study contributes to a growing literature about earnings management in the public sector, helping to identify circumstances that can lead to opportunistic behavior by public managers, especially in relation to elections.

Keywords: earnings management; electoral cycle; municipalities. 


\section{INTRODUÇÃO}

A aplicação do regime de competência (accrual basis) visa promover informação incremental ao fluxo de caixa. Mas, em virtude de problemas de conflitos de interesse, as escolhas contábeis também podem ser utilizadas para o gerenciamento de resultados (Dechow, Sloan \& Sweeney, 1995). No setor privado, pesquisas já identificaram determinantes do gerenciamento, como tamanho, tipo de firma de auditoria, estrutura de governança corporativa etc. - para uma revisão, vide, por exemplo, Dechow, Ge e Schrand (2010).

No setor público, existe um movimento de ampliação da utilização do regime de competência, que também permitiria que políticos pudessem exercer sua influência para gerenciar resultados, tendo em vista a tentativa, por parte dos gestores públicos, de controlar as percepções e as avaliações de suas condutas pelos cidadãos (Leary \& Kowalski, 1990).

A título de exemplo, em municípios portugueses, há evidências de que políticos locais realizam escolhas contábeis para evitar divulgar resultados deficitários em épocas de eleição (Ferreira, Carvalho \& Pinho, 2013). Mesmo diante desse cenário, estudos no setor público sobre gerenciamento de resultados ainda são relativamente escassos (Ramos \& Costa, 2011).

No cenário público brasileiro atual, os governos federal, regionais e locais buscam a harmonização com as Normas Internacionais de Contabilidade Aplicadas ao Setor Público (Ipsas), a qual prevê a disseminação dessas normas, o intercâmbio de experiências e o fomento de iniciativas que visem às melhores práticas internacionais de contabilidade aplicada ao setor público (Portaria no 548 de 24 de setembro de 2015). Diante disso, municípios passaram a adotar, paulatinamente, novos regramentos com base no regime de competência.

Pesquisas apontam para um comportamento oportunista de gestores públicos em benefício próprio, sobretudo em períodos eleitorais, na busca pela maximização de votos para reeleição (Akhmedov \& Zhuravskaya, 2004; Baber \& Sen, 1986; Buchanan \& Tullock, 1962; Coelho, 2004; Drazen \& Eslava, 2005; Nakaguma \& Brender, 2006; Preussler \& Portugal, 2003). Por exemplo, há evidências de que indivíduos e partidos que estão no poder manipulam políticas públicas (Baber \& Sen, 1986) e ampliam transferências de renda diretas para eleitores (Akhmedov \& Zhuravskaya, 2004).

Neste trabalho, a alteração de políticas, com vistas ao aumento do sucesso nas eleições, é definida como comportamento oportunista de um gestor público. Assim, o cenário de adoção do regime de competência no Brasil gera um contexto oportuno para estudar a atuação dos gestores públicos num ambiente de diversidade de tipos de cidades e suas características, o que pode contribuir para a adequada compreensão desse fenômeno.

Diante do exposto nos parágrafos anteriores, o propósito desta pesquisa é investigar se há relação entre o aumento nos níveis de gerenciamento de resultados em municípios brasileiros e os períodos pré-eleitorais e eleitorais.

Ao usar o modelo de Burgstahler e Dichev (1997) na análise de distribuição de frequências, constatou-se uma descontinuidade em torno do resultado nulo (breakeven), bem como sua relação positiva com os níveis de gerenciamento de resultados, estimado por meio dos accruals discricionários, com base no modelo Jones modificado (1995). Posteriormente, verificou-se a relação positiva entre o gerenciamento de resultados e os períodos pré-eleitoral e eleitoral, assim como a relação negativa entre a prática de gerenciamento e o ambiente de competição política. De maneira geral, há evidências de que os municípios implementaram práticas de gerenciamento de resultados em função do ciclo eleitoral.

O presente trabalho pretende contribuir para suprir a falta de informação sobre o tema no setor público, bem como para a identificação de padrões de comportamento dos gestores locais brasileiros 
e sua inter-relação com os ciclos eleitorais. Além disso, intermediários financeiros, como o controle externo, na forma de Tribunais de Contas ou Auditorias Gerais, poderão concentrar esforços em auditorias específicas com base em critérios de risco sobre eventos de gerenciamento de resultados, ampliando o grau de confiança nas demonstrações financeiras apresentadas e, consequentemente, reduzindo a assimetria informacional e melhorando as características qualitativas da informação contábil apresentada pelos relatórios financeiros governamentais.

O próximo capítulo traz o referencial teórico acerca do tema, explicando o desenvolvimento da contabilidade pública no Brasil e no âmbito internacional em seus aspectos mais significativos, o gerenciamento de resultados e sua relação com o ciclo eleitoral. Depois, é abordada a metodologia utilizada no trabalho, com a descrição das variáveis e as hipóteses desenvolvidas. Por último, são apresentados os resultados e as conclusões das análises empíricas.

\section{REFERENCIAL TEÓRICO}

\subsection{Contabilidade Pública: Desenvolvimento no Âmbito Internacional e Nacional}

No contexto da nova gestão pública, que introduz o paradigma na avaliação do desempenho dos gestores públicos, o resultado divulgado pelo regime de competência se torna um importante indicador de eficiência e eficácia para políticas locais que buscam atender às necessidades dos cidadãos (Ferreira et al., 2013).

A chamada "contabilidade pública tradicional", utilizada até o fim dos anos 1980, tinha foco no regime de caixa e se preocupava, basicamente, em controlar a execução do orçamento, buscando garantir o controle efetivo dos recursos públicos (García, 2014). As transações eram registradas no momento do recebimento ou pagamento, não fornecendo informações necessárias à correta tomada de decisão por parte dos gestores (García, 2014).

Com isso, fatores como a globalização, o crescimento da importância do déficit público, a concorrência aberta entre a área pública e a área privada no fornecimento de serviços específicos, a pressão por maior transparência dos relatórios públicos e a necessidade política de responder ao eleitorado com serviços públicos orientados ao cidadão-cliente iniciaram o movimento na direção da modernização da contabilidade do setor público (García, 2014). Desta forma, busca-se uma abordagem voltada para eficiência e eficácia, simplificação da burocracia e divulgação de informações a terceiros, além do maior controle sobre as despesas e flexibilidade nas tomadas de decisões, culminando em uma revisão de toda estrutura contábil do setor público, modelada pela padronização aplicada ao setor privado (García, 2014, p. 349).

Desde então, o International Public Sector Accounting Standards Board (Ipsasb), da International Federation of Accountants (Ifac), vem desenvolvendo as Ipsas, promovendo contínuas mudanças no desenvolvimento de uma nova abordagem, evoluindo gradualmente de modo a demonstrar os aspectos econômicos, financeiros e patrimoniais das atividades do setor público (García, 2014). Entre as alterações, o princípio da competência se insere como um grande passo para que as demonstrações financeiras apresentem uma visão real das atividades econômicas das entidades públicas (Barea, 1994).

Espera-se que o regime de competência possa auxiliar na avaliação e no monitoramento dos gestores, assim como da transparência (International Monetary Fund [IMF], 2014). Não obstante, são fatores que contribuem para a qualidade da informação: controles internos, auditoria externa e

REVISTA DE AdMINIStração PÚblica ｜ Rio de Janeiro 55(3): 697-715, maio - jun. 2021 
um Poder Legislativo com habilidade de exigir do Executivo a contabilização para que tal reforma ocorra de maneira adequada (Hepworth, 2003).

No que tange ao Brasil, a busca por informações com maior transparência e comparabilidade, impulsionada pela expansão de mercados, levou à decisão de iniciar o processo de adoção de um novo modelo contábil para o setor público, baseado no "modelo internacional" (Silva, 2012). Dessa forma, o processo de convergência aos padrões internacionais teve início em 2008, quando o Conselho Federal de Contabilidade (CFC) e a Ifac firmaram um compromisso institucional, culminando nas primeiras Normas Brasileiras de Contabilidade (NBC TSP 16), bem como nas edições periódicas do Manual de Contabilidade Aplicada ao Setor Público (Mota, 2015).

Ainda em 2008, foi publicada a Portaria MF no 184/2008, que dispõe sobre as diretrizes a serem observadas pelos entes na busca pela convergência (Portaria $\mathrm{n}^{\circ} 184$ de 25 de agosto de 2008). Com isso, a contabilidade pública brasileira, que nas últimas 5 décadas manteve seu foco nos aspectos orçamentários por conta das regras bem-definidas de reconhecimento esculpidas na Lei Federal no 4.320/1964, passa a buscar o papel de tratamento contábil com toda a plenitude científica, tanto para os aspectos orçamentários quanto para os patrimoniais, que deveriam ser registrados por competência (Mota, 2015).

Como continuidade do processo, a Secretaria do Tesouro Nacional editou, em 2015, a Portaria $n^{\circ}$ 548. Nela, estão definidos os prazos para implantação dos procedimentos contábeis patrimoniais na federação brasileira com base na experiência nacional e internacional, de forma que o gradualismo na implantação siga uma lógica estabelecida com a observação de critérios bem-definidos e devidamente contextualizados (Portaria no 548 de 24 de setembro de 2015). Esse normativo trouxe, após ampla discussão com os representantes da união, dos estados e dos municípios, os prazos para adoção do regime de competência nas mais diversas transações do setor público, como reconhecimento, evidenciação e mensuração de obrigações com fornecedores, provisões, imobilizado, créditos tributários, entre outros (Portaria no 548 de 24 de setembro de 2015).

No fim de 2016, o processo de convergência no país foi intensificado com a edição de diversas Normas Brasileiras de Contabilidade, em substituição às NBC TSP 16, com destaque para a aprovação da nova Estrutura Conceitual (NBC TSP EC), a ser utilizada na elaboração e na divulgação da informação contábil de propósito geral pelas entidades do setor público (CFC, 2016). Surge o conceito de Relatórios Contábeis de Propósito Geral das Entidades do Setor Público (RCPGs), os quais devem ser elaborados com base no regime de competência (CFC, 2016). Tais relatórios devem fornecer informações úteis aos seus usuários, de modo a servir como base, mesmo que parcialmente, para a prestação de contas e responsabilização (accountability) e tomada de decisão (CFC, 2016).

De acordo com o Ipsasb (2016), a contabilidade aplicada ao setor público é baseada nos padrões internacionais aplicados ao setor privado (IFRS). Assim, os critérios de reconhecimento, evidenciação e mensuração permitem que modelos científicos de estudos aplicados na iniciativa privada se mostrem eficientes em pesquisas no setor público (Ferreira et al., 2013).

\subsection{Gerenciamento de Resultados}

Gerenciamento de resultados (earnings management) se apresenta quando os gestores se utilizam de julgamentos nas demonstrações financeiras e nas estruturas das transações para levar as partes interessadas a um entendimento distorcido do desempenho econômico de uma entidade. Tais julgamentos podem ser feitos de diversas maneiras, como na estruturação de transações corporativas, na expectativa de recuperação de ativos de longo prazo, na escolha no método de depreciação, em

ReVISTA de Administração PÚblica ｜ Rio de Janeiro 55(3): 697-715, maio - jun. 2021 
obrigações de benefícios de pensão e na realização ou no adiamento de determinada despesa (Healy \& Wahlen, 1999).

O raciocínio parte da visão contábil tradicional de que os registros por competência são mais fáceis de ser manipulados do que o fluxo de caixa por conta das estimativas e das provisões utilizadas em sua mensuração (Dechow, Richardson \& Tuna, 2003). Essa flexibilização pode ser utilizada pelos gestores para alcançar seus objetivos.

O ponto inicial de partida para mensurar o gerenciamento de resultados se baseia na apuração dos accruals totais, em que um modelo particular é assumido para possibilitar sua decomposição nos componentes discricionários e não discricionários (Dechow et al., 1995). Entende-se por accruals a diferença entre o resultado apurado pelo regime de competência e o fluxo de caixa das operações durante determinado período (Dechow et al., 2010).

Partindo da análise da qualidade do resultado com base nos accruals demonstrados pelas entidades, diversas pesquisas buscaram distinguir os accruals "não discricionários" dos "discricionários" por meio de modelagem direta, haja vista que estes são utilizados como proxy na captura de distorções induzidas pelo gerenciamento (Dechow et al., 2010).

Dos diversos modelos desenvolvidos e largamente utilizados no meio acadêmico (Dechow et al., 1995; Dechow \& Dichev, 2002; Francis et al., 2005; Jones, 1991; Kothari et al., 2005), Dechow et al. (2010) destacam que seus potenciais em identificar os accruals "anormais" devem levar em conta a possibilidade de erros de tipo I, que classificam os accruals como "anormais" quando, na verdade, eles representam a performance da entidade, e dos erros de tipo II, que classificam os accruals como "normais" quando eles não são.

O modelo Jones modificado (1995) vem sendo o mais empregado na identificação de gerenciamento de resultados, sendo amplamente utilizado nas pesquisas acadêmicas (Dechow et al., 1995; Ferreira \& Costa, 2011). O modelo deriva de Jones (1991), que já se diferenciava dos demais por relaxar a premissa de que os accruals não discricionários são constantes, buscando controlar os efeitos de mudanças nas circunstâncias econômicas da entidade analisada sobre os accruals discricionários (Dechow et al., 1995). No modelo modificado, parte-se da premissa de que a variação dos créditos de curto prazo são accruals discricionários, ampliando o poder de detecção de gerenciamento (Dechow et al., 1995).

\subsubsection{Gerenciamento de resultados e ciclos eleitorais}

Para Przeworski et al. (1999), a relação entre políticos e cidadãos pode ser examinada pela perspectiva da teoria da agência, que surge quando o agente deve agir em favor do principal, com vistas a implementar as preferências deste. No mundo político, os cidadãos, por meio do voto, elegem um candidato com base em programas eleitorais e promessas de campanha mais adequados às suas preferências, criando a expectativa de que o agente eleito, agora investido de poder, responda a seu eleitorado implementando tais preferências.

Políticos, no entanto, tendem a agir de forma oportunista com vistas a ganhar eleições e se manter no poder mesmo quando suas políticas divergem das preferências dos eleitores. Nesses casos, chamados de conflito de agência, os agentes podem fazer uso de estratégias manipuladoras para evitar o custo de tais divergências. Com isso, ações oportunistas geram assimetrias de informação e, consequentemente, problemas de accountability, retrospectivamente por "risco moral", na medida em que as informações fornecidas pelo agente não forem verificáveis, limitando o poder do eleitor em recompensar os bons agentes e punir os oportunistas pelo voto (Przeworski et al., 1999). 
Aplicando-se essa teoria ao contexto contábil, espera-se que os agentes políticos usem informações contábeis como forma de demonstrar seu desempenho, ao passo que o principal - formado por usuários dos serviços, fornecedores de recursos e seus representantes - se baseie nessas informações no monitoramento das ações políticas e no estabelecimento dos termos contratuais entre as partes (Zimmerman, 1977).

Conflitos de interesse entre o principal e os agentes apontam para a existência de motivação suficiente para a ocorrência de gerenciamento de resultados no setor público (Ferreira et al., 2013). Corroborando esse entendimento, Wynne (2007) ressalta que o movimento na direção da accrual accounting no setor público traz consigo o risco de que decisões no tratamento contábil sejam tomadas com base em motivos políticos. Tal fato tem relação direta com a abordagem utilizada pela contabilidade na adoção de suas normas. No entanto, de acordo com a European Federation of Accountants (2003), esse efeito pode ser atenuado ou evitado por precondições adotadas pelos governos durante a implementação, como a inserção de auditores externos no processo de convergência, o fortalecimento de uma cultura ética internalizada e de um sistema de controle aceito e entendido entre os diversos departamentos, um processo de auditoria externa robusto com vistas a suprir o parlamento de informações sobre as demonstrações financeiras e a inexistência de corrupção sistemática.

A utilização de padrões contábeis baseados em princípios (principle-based accounting standards) forneceria margem para julgamentos por parte dos envolvidos na busca pela divulgação de contextos econômicos específicos - e, consequentemente, maior possibilidade de comportamentos oportunistas -, ao passo que a adoção de padrões contábeis baseados em regras (rules-based accounting standards) restringe substancialmente tais escolhas (Kothari, Ramana \& Skinner, 2010).

Entre os estudos realizados sobre o tema de ciclos políticos e eleitorais, Akhmedov e Zhuravskaya (2004), com base em dados da Rússia, confirmaram o aumento de gastos em período pré-eleitoral em torno de $5 \%$ em programas de bem-estar e assistência, bem como que a manipulação no mesmo período aumenta a chance de reeleição. No Brasil, Gonçalves, Funchal e Bezerra (2017) demonstraram que o ciclo eleitoral, compreendendo os anos eleitorais e pré-eleitorais, influenciam nos investimentos com infraestrutura dos estados.

Ferreira et al. (2013), ao analisarem as municipalidades de Portugal, demonstraram evidências de que políticos locais gerenciam resultados com o objetivo de evitar os negativos e divulgar os positivos. Dessa forma, os resultados divulgados não serão interpretados por cidadãos, grupos de interesse ou partidos políticos como excessivo. A pesquisa também evidenciou que ambientes de forte competição política mostraram menor predisposição para evitar divulgar resultados negativos (Ferreira et al., 2013).

\subsection{Hipóteses}

A primeira abordagem utilizada neste trabalho para a detecção de gerenciamento de resultados, denominada distribuição de frequências, busca detectar eventuais descontinuidades por meio da análise de histograma em torno do resultado "zero" (breakeven), indicando possível gerenciamento de resultados para evitar pequenos prejuízos (Burgstahler \& Dichev, 1997). Assim, a seguinte hipótese alternativa foi desenvolvida: H1 - Há descontinuidade na distribuição dos resultados gerados pelos municípios em torno do zero. 
Espera-se que a hipótese alternativa se confirme, indicando uma descontinuidade em torno do resultado nulo, com maior parte dos municípios apresentando pequenos resultados positivos, com o objetivo de demonstrar eficiência ao usuário da informação (Ferreira \& Costa, 2011; Pilcher \& Zahn, 2008).

Não obstante, deve ser levado em consideração o fato de que uma eventual descontinuidade identificada na distribuição de frequências pode ter sido ocasionada por ações reais que levaram a uma melhora no desempenho da entidade (Dechow et al., 2003). Logo, foi elaborada a seguinte hipótese alternativa: $\mathbf{H 2}$ - O nível de gerenciamento de resultados é maior para municípios que apresentam pequenos resultados positivos em comparação aos que apresentam pequenos resultados negativos.

A expectativa é a de que a hipótese alternativa se confirme, indicando uma relação positiva entre o nível de gerenciamento de resultados e os municípios que apresentaram pequenos resultados positivos.

Por fim, buscou-se evidenciar o gerenciamento de resultados em períodos pré-eleitorais e eleitorais por parte dos políticos locais com o objetivo de demonstrar maior eficiência na gestão pública. Para isso, foi desenvolvida a seguinte hipótese alternativa: H3 - O nível de gerenciamento de resultados é maior em períodos pré-eleitorais em comparação com os demais.

Tendo em vista a literatura sobre o tema, abordada na sessão 2.2.1, espera-se que haja uma relação positiva entre o período pré-eleitoral/eleitoral e os níveis de accruals discricionários, indicando que tais resultados sofreram influência de gerenciamento de resultados para serem alcançados.

Adicionalmente, foi introduzida uma variável de mensuração de competição política que procura demonstrar o impacto negativo no gerenciamento de resultados segundo a teoria da escolha pública, uma vez que um ambiente de forte competição política será mais monitorado por parte de cidadãos, grupos de interesse e partidos políticos, inibindo práticas oportunistas dos gestores atuais, que tendem a evitar a perda de votos destinados à reeleição (Ferreira et al., 2013). Assim, há a expectativa de uma relação negativa entre o gerenciamento de resultados e a competição política.

\section{METODOLOGIA}

\subsection{Amostra}

A amostra consiste nas declarações de contas anuais (DCAs) dos municípios brasileiros que encaminharam seus saldos contábeis ao Ministério da Fazenda por meio do Sistema de Informações Contábeis e Fiscais do Setor Público Brasileiro (Siconfi), no período de 2013 a 2016. A pesquisa tem também como base as informações relativas ao ano de 2016 contidas no Tribunal Superior Eleitoral (TSE) sobre o resultado das eleições, a fim de verificar se o ambiente competitivo impacta no nível de gerenciamento de resultados.

O período escolhido procurou manter um ciclo eleitoral para escolha dos governantes locais, objetivando identificar maior incidência de gerenciamento no exercício pré-eleitoral (2015), haja vista que esse período pode influenciar os investimentos em infraestrutura (Gonçalves et al., 2017). A amostra inicial totaliza 18.523 observações, distribuídas, como mostra a Tabela 1, ao longo dos anos. 
TABELA 1 TOTAL DE OBSERVAÇÕES POR EXERCíCIO FINANCEIRO

\begin{tabular}{|cc|}
\hline EXERCÍCIO FINANCEIRO & No DE OBSERVAÇÕES \\
2013 & 2.624 \\
2014 & 5.182 \\
2015 & 5.401 \\
2016 & 5.316 \\
\hline
\end{tabular}

Fonte: Dados da pesquisa.

Como é possível perceber na Tabela 1, o ano de 2013 apresentou um número significativamente inferior de observações quando comparado aos demais, o que pode ser considerado uma limitação do trabalho. Isso se deve ao fato de que o envio de dados ao Siconfi nesse exercício ainda era facultativo, tornando-se obrigatório apenas a partir de 2014.

Após a coleta dos dados, foram selecionadas, por meio das contas patrimoniais e de resultados, as variáveis necessárias à apuração dos accruals discricionários. Todas as variáveis contínuas utilizadas nos modelos desta pesquisa foram tratadas para mitigar o efeito de outliers. Foi empregada a técnica Winsor em $1 \%$ em cada cauda da distribuição da amostra.

\subsection{Modelos da Pesquisa}

Para testar a hipótese 1, foi utilizado o modelo de medida de significância de estatística Z, elaborado por Burgstahler e Dichev (1997). O teste parte da premissa de que a distribuição de frequência dos resultados apresentados deve ser normal. Dessa forma, foi utilizada a seguinte equação:

$$
Z=\frac{n a_{i}-n e_{i}}{\sigma\left(n a_{i}-n e_{i}\right)}
$$

Em que é o número real de observações no intervalo $i$; $n e_{i}$ é o número esperado de observações no intervalo $i$, obtido da média entre o intervalo imediatamente anterior e o intervalo imediatamente posterior ao intervalo $i$; e $\sigma\left(n a_{i}-n e_{i}\right)$ é o desvio-padrão da diferença entre o número real e o número esperado para o intervalo $i$. Para apurar o valor de $\sigma$, utilizou-se a seguinte equação:

$$
\sigma=\sqrt{N p_{i}\left(1-p_{i}\right)+\frac{N\left(p_{i-1}+p_{i+1}\right)\left(1-p_{i-1}-p_{i+1}\right)}{4}}
$$

Em que $\mathrm{N}$ representa o número total de observações da amostra; $p_{i}$ é a probabilidade de que uma observação esteja localizada no intervalo $i$; e $p_{i-1}$ e $p_{i+1}$ são as probabilidades de uma observação estar localizada nos intervalos anterior e posterior, respectivamente.

Os testes empíricos realizados para testar a hipótese 2 se baseiam no modelo Jones modificado (Dechow et al., 1995), também empregado no setor público, após ajustes, conforme Ferreira et al. (2013). Para o cálculo do total de accruals, foi empregado o método indireto (Healy, 1985; Jones, 1991), conforme apresentado a seguir: 


$$
T A_{i t}=\left[\left(\Delta A C_{i t}-\Delta D i s p_{i t}\right)-\left(\Delta P C_{i t}-\Delta D I V\right)-\text { Deprecia }_{i t}\right]
$$

Em que $T A_{i t}$ representa o total de accruals para o município $i$ no exercício $t ; \Delta A C_{i t}$ é a variação do ativo circulante do município $i$ entre o período $t$ e $t-1 ; \Delta D I S P_{i t}$ é a variação do caixa do município $i$ entre o período $t ; \Delta D I V_{i t}$ é a variação de empréstimos e financiamentos de curto prazo do município $i$ entre o período $t$ e $t-1 ; \Delta P C_{i t}$ é a variação do passivo circulante do município $i$ entre o período $t$ e $t$-1; e Deprecia ${ }_{i t}$ é a soma das VPDs (despesas por competência) com depreciação, amortização e exaustão apuradas no município $i$ no período $t$.

Após o cálculo do total de accruals, escalonado pelos ativos totais, foi rodada a seguinte regressão para estimar os accruals não discricionários, com base no modelo Jones modificado (Dechow et. al.,1995).

$$
T A_{i t}=a_{1}\left(1 / A_{i t-1}\right)+\beta_{1}\left(\Delta R E V_{i t}-\Delta R E C_{i t}\right)+\beta_{2}\left(P P E_{i t}\right)+\varepsilon_{i t}
$$

Em que $A_{t-1}$ é o ativo total da entidade $i$ no período $t-1 ; \Delta R E V_{t}$ são as receitas brutas obtidas pela entidade $i$ no ano $t$ menos a receita bruta obtida no ano $t$-1, escalonados pelo ativo total da entidade no período $t-1 ; \triangle R E C_{t}$ representa os créditos a receber de curto prazo da entidade $i$ no ano $t$ menos os créditos a receber de curto prazo no ano t-1; e $P P E_{t}$ são os ativos imobilizados da entidade $i$ no período $t$, escalonados pelo ativo total em $t-1$.

Os resíduos do modelo foram utilizados como proxy para o nível de gerenciamento de resultados, medido por meio dos accruals discricionários (DA). No setor privado, em geral a equação é rodada para cada setor/ano. No caso do setor público, optou-se por rodar a regressão segregada por porte de municípios. Nesse caso, os municípios foram divididos por decil, com base em sua população. A equação foi, então, estimada para cada decil, permitindo a obtenção dos resíduos. Assim, espera-se que a estimação seja realizada com base em municípios de caraterísticas semelhantes.

Por fim, de posse dos accruals discricionários estimados pela equação 4, utilizou-se o seguinte modelo Probit para apurar se há relação entre gerenciamento de resultados e os municípios que divulgaram pequenos resultados positivos (Ferreira et al., 2013):

$$
\text { Inter }_{i t}=a_{i t}+\beta_{1}\left(D A_{i t}\right)+\text { CONTROLES }+\varepsilon_{i t}
$$

Em que representa a variável dummy que divide os municípios em 2 grupos: os que apresentaram "pequenos resultados negativos" (Inter=0) e os que apresentaram "pequenos resultados positivos" (Inter=1). Como variáveis de controle, foram inseridas dummies para cada período e estado.

Para testar a hipótese 3, elaborou-se um modelo de regressão inspirado em Ferreira et al. (2013), com o objetivo de verificar se os accruals discricionários são influenciados pela competição política e pelos períodos pré-eleitorais. Para isso, foram testadas as seguintes equações:

$$
D A_{i t}=\alpha_{i t}+\beta_{1}\left(\text { Ieleição }_{i t}\right)+\beta_{2}\left(\text { Dcomp }_{t+1}\right)+\varepsilon_{i t}
$$

Em que são os accruals discricionários estimados pelo modelo Jones modificado (1995); é a variável dummy que particiona a amostra entre os períodos pré-eleitoral e eleitoral (2015 e 2016) e os 
demais períodos (2014); é a variável dummy que particiona a amostra entre os grupos que apresentam ambiente de alta competitividade (mais de 2 candidatos disputando o cargo) e menor competitividade ( 2 ou menos candidatos disputando o cargo) no ano eleitoral.

A apuração da variável Dcomp foi obtida pelo modelo criado por Laakso e Taagepera (1979), que busca demonstrar o efetivo número de partidos de acordo com o percentual aferido de votos durante as eleições. Tal modelo é utilizado para verificar o número efetivo de partidos políticos numa eleição (Ferreira et al., 2013), sendo uma medida confiável calculada por meio da seguinte equação:

$$
\operatorname{Comp}=\frac{1}{\sum_{i=1}^{n} p_{i}^{2}}
$$

Em que $p_{i}^{2}$ é a parte fracionária de votos obtidos durante a eleição para o partido $i$.

Na próxima seção são apresentados e discutidos os resultados empíricos do trabalho.

\section{RESULTADOS}

\subsection{Estatística Descritiva}

A Tabela 2 apresenta a estatística descritiva para as variáveis utilizadas no presente trabalho.

\section{TABELA 2 ESTATÍSTICA DESCRITIVA DAS VARIÁVEIS UTILIZADAS}

\begin{tabular}{ccccccc}
\multicolumn{7}{c}{ Painel A: Estatística descritiva das variáveis } \\
Variáveis & $\mathbf{N}$ & Média & Mediana & Desv. Padrão & min. & max. \\
leleicao & 10.093 & 0,816 & 1 & 0,388 & 0 & 1 \\
Dcomp & 10.093 & 0,159 & 0 & 0,366 & 0 & 1 \\
TA & 10,431 & $-0,0$ & 0,0 & 0,186 & $-0,833$ & 0,7 \\
1/A t-1 & 10,431 & 0,01 & 4,77 & 0,980 & $-9,75$ & 100 \\
$\Delta R E V-\Delta R E C$ & 10,431 & 0,258 & 0,133 & 0,742 & $-18,86$ & 42,27 \\
PPE & 10,431 & 0,778 & 0,805 & 0,323 & 0,01 & 23,36 \\
DA & 10.093 & 0,008 & 0,006 & 0,183 & $-0,864$ & 0,793 \\
Inter & 2038 & 0,7 & 1 & 0,458 & 0 & 1
\end{tabular}

Ieleição é a variável dummy que particiona a amostra entre os períodos pré-eleitoral e eleitoral (2015 e 2016) e os demais períodos (2013 e 2014); Dcomp é a variável dummy que particiona a amostra entre os grupos que apresentam ambiente de alta competitividade (mais de 2 candidatos disputando o cargo) e menor competitividade ( 2 ou menos candidatos disputando o cargo) no ano eleitoral, calculada pelo seguinte modelo de Laakso e Taagepera (1979) $\operatorname{Comp}=\frac{1}{\sum_{i=1}^{n} p_{j}^{2}}$; TA demonstra o total de accruals calculado pela equação $T A_{i t}=\left[\left(\Delta A C_{i t}-\Delta D i s p_{i t}\right)-\left(\Delta L C_{i t}-\Delta D I V\right)-\right.$ Deprecia $\left._{\mathrm{it}}\right] ; A t-1$ é o ativo total no período t-1; $\Delta \mathrm{REV}_{\mathrm{it}}$ ão as receitas brutas obtidas pelas entidades no ano $t$ menos a receita bruta obtida no ano $\mathrm{t}-1, \Delta \mathrm{REC}_{\mathrm{it}}$ escalonados pelo ativo total da entidade no período $\mathrm{t}-1$; representa os créditos a receber de curto prazo das entidades no ano $t$ menos os créditos a receber de curto prazo no ano $t-1$, escalonados pelo ativo total da entidade no período t-1; PPE são os ativos imobilizados das entidades no período $t$, escalonados pelo ativo total em $t$ - 1 ; $D A_{i t}$ são os accruals discricionários calculados pelo modelo Jones modificado (Dechow et al., 1995), dado pela seguinte regressão:

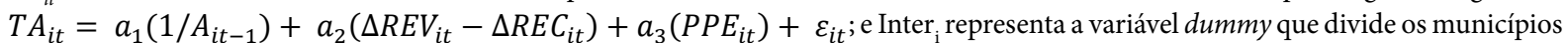
entre aqueles que apresentaram pequenos resultados negativos (Inter $=0$ ) e aqueles que apresentaram pequenos resultados positivos (Inter=1).

Fonte: Elaborada pelos autores. 
O número de observações das variáveis Ieleicao e Dcomp é ligeiramente inferior às demais variáveis. Tal fato se deve à ausência de dados eleitorais para alguns municípios no exercício de 2016 . A variável Inter também tem um número inferior de observações por apresentar apenas aqueles municípios que demonstraram pequenos resultados positivos ou negativos.

\subsection{Resultados do Teste de Hipótese H1}

Em relação à hipótese 1, foi gerado um histograma para análise da distribuição de frequência dos resultados divulgados pelos municípios brasileiros no período de 2013 a 2016, com o objetivo de identificar uma descontinuidade em torno do breakeven. O resultado está demonstrado na figura 1.

\section{FIGURA 1 DISTRIBUIÇÃO DE FREQUÊNCIA DA VARIÁVEL "RESULTADO"}

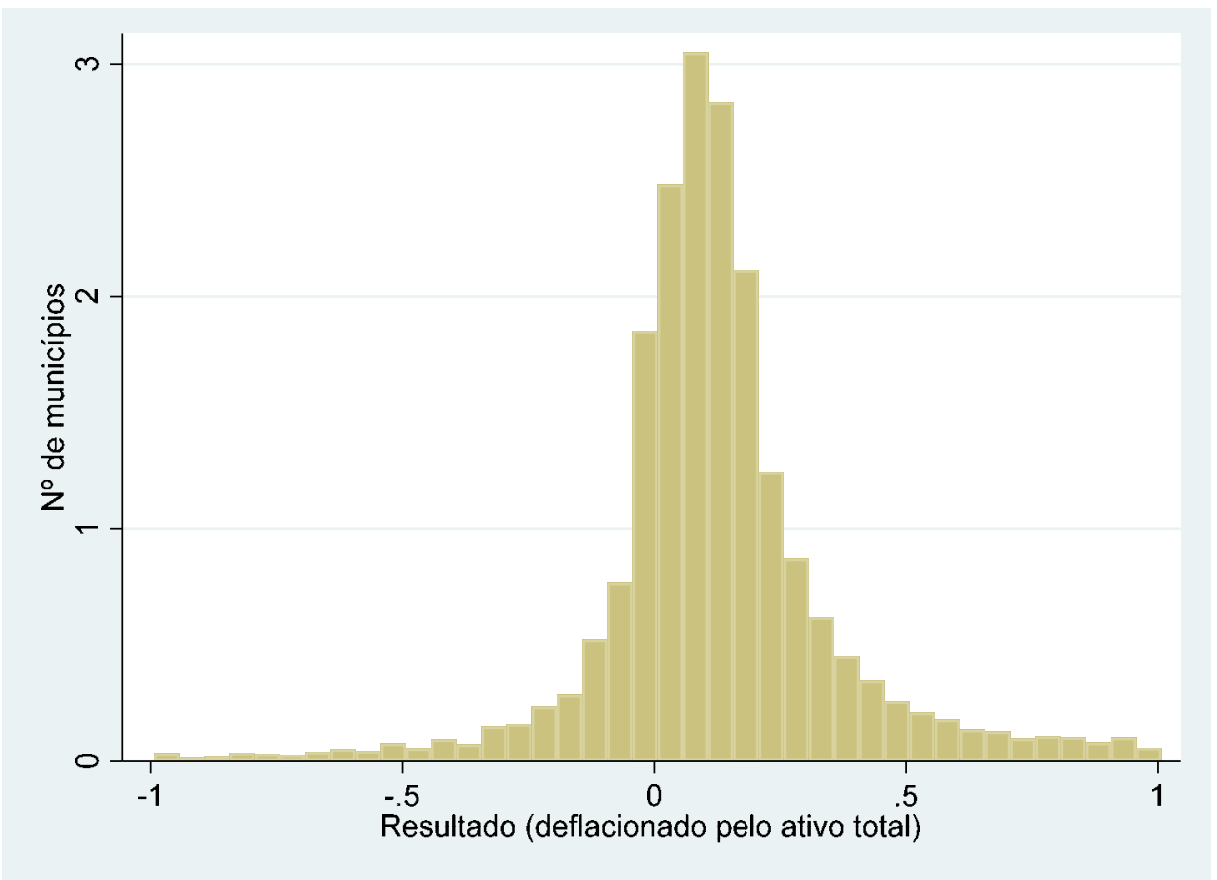

Fonte: Elaborada pelos autores.

Com base no histograma, é possível perceber que há uma descontinuidade aparente entre o intervalo composto por municípios que apresentaram pequenos resultados negativos (611 municípios) e o intervalo composto por municípios que apresentaram pequenos resultados positivos (1.427 municípios). As frequências de observações são apresentadas na Tabela 3. 
TABELA 3 INTERVALOS DO HISTOGRAMA

\begin{tabular}{c|cc} 
VARIÁVEL INTERVAL & INTERVAL0 & OBSERVAÇÕES \\
-3 & $j-0,15 ;-0,10]$ & 243 \\
-2 & $j-0,10 ;-0,05]$ & 360 \\
-1 & $j-0,05 ; 0,00]$ & 611 \\
0 & $j 0,00 ; 0,05]$ & 1,427 \\
1 & $j 0,05 ; 0,10]$ & 1,503 \\
2 & $j 0,10 ; 0,15]$ & 1,45 \\
Total de observações da amostra & 10.431
\end{tabular}

Fonte: Elaborada pelos autores.

Após aplicação das equações 1 e 2 apresentadas na sessão 3.2, verificou-se que a descontinuidade apontada graficamente é significante em níveis confiáveis, rejeitando a hipótese nula. O resultado pode ser mais bem analisado na Tabela 4 .

\section{TABELA 4 SIGNIFICÂNCIA ESTATÍSTICA DAS DESCONTINUIDADES PRÓXIMAS AO ZERO DA DISTRIBUIÇÃO DE FREQUÊNCIAS DO RESULTADO DO EXERCÍCIO}

\begin{tabular}{|cccc|}
\hline INTERVAL0 & $\begin{array}{c}\text { Número de } \\
\text { observações reais }\end{array}$ & $\begin{array}{c}\text { Número de } \\
\text { observações esperadas }\end{array}$ & $\begin{array}{c}\text { Estatística Z } \\
\text { (p-value) }\end{array}$ \\
\hline$[-0,05 ; 0,00]$ & 611 & 893,5 & $-6,95^{\star \star \star}$ \\
{$[0,00 ; 0,05]$} & 1.427 & 1057 & $12,03^{\star \star \star}$ \\
\hline
\end{tabular}

O teste de significância e o número de observações esperadas foram calculados pelo modelo de Burgstahler e Dichev (1997), que parte da premissa de que a distribuição de frequência dos resultados apresentados deve ser normal, ou seja, deve apresentar assimetria próxima à nula $(\mathrm{Z}=0)$. O cálculo é feito pela seguinte equação: $Z=\frac{n a_{i}-n e_{i}}{\sigma\left(n a_{i}-n e_{i}\right)}$, em que $n a_{i}$ é o número real de observações no intervalo $i$; $n e_{i}$ é o número esperado de observações no intervalo $i$, obtido da média entre os intervalos anterior e posterior $i$; e $\sigma\left(n a_{i}-n e_{i}\right)$ é o desvio-padrão da diferença entre o número real e o número esperado para o intervalo $i$. Para apurar o valor de $\sigma$, utilizou-se a seguinte equação: $\sigma=\sqrt{N p_{i}\left(1-p_{i}\right)+\frac{N\left(p_{i-1}+p_{i+1}\right)\left(1-p_{i-1}-p_{i+1}\right)}{4}}$, em que $\mathrm{N}$ representa o número total de observações da amostra; $p_{i}$ é a probabilidade de que uma observação esteja localizada no intervalo $i$; e $p_{i-1}$ e $p_{i+1}$ são as probabilidades de uma observação estar localizada nos intervalos anterior e posterior, respectivamente.

Fonte: Elaborada pelos autores.

Logo, há evidências significativas de uma descontinuidade na distribuição dos resultados gerados pelos municípios em torno do zero, com um número maior que o esperado de municípios apresentando pequenos resultados positivos e um número menor apresentando pequenos resultados negativos. 


\subsection{Resultados do Teste de Hipótese H2}

Com relação ao teste da hipótese 2, foi utilizado o modelo Jones modificado (1995), conforme descrito na sessão 3.2 (Equação 4), com o objetivo de verificar se há relação entre a prática de gerenciamento de resultados e a divulgação de pequenos resultados positivos. A Tabela 5 apresenta as estatísticas descritivas e os coeficientes obtidos por meio da equação 7 para as variáveis usadas para testar a hipótese 2 (Inter e DA) dos 2.038 municípios-ano que divulgaram resultados próximos ao zero (-0,05; 0,05) em suas Declarações Anuais de Contas, nos exercícios financeiros de 2014 a 2016, resultando nos dados apresentados abaixo.

\section{TABELA 5 COEFICIENTES DO MODELO DE PROBIT UTILIZADO NA HIPÓTESE 2}

\begin{tabular}{|c|c|c|c|c|c|}
\hline \multicolumn{6}{|c|}{ Painel A: Estimativa dos coeficientes obtidos através do modelo de Probit } \\
\hline Variáveis & $\mathbf{N}$ & Coeficientes & Erro padrão & Estatística Z & p-value \\
\hline Constante & 2.038 & 0,025 & 0,887 & 0.03 & 0,978 \\
\hline$D A$ & 2.038 & 0,417 & 0,179 & 2.33 & 0,020 \\
\hline CONTROLES & Sim & & & & \\
\hline \multicolumn{6}{|c|}{ Painel B: Coeficiente de determinação } \\
\hline$R 2$ & & 0,033 & & & \\
\hline
\end{tabular}

A Probit foi calculada utilizando o seguinte modelo: Inter $_{i t}=a_{i t}+\beta_{1}\left(D A_{i t}\right)+\varepsilon_{i t}$, em que Inter ${ }_{i t}$ representa a variável dummy que divide os municípios entre aqueles que apresentaram pequenos resultados negativos (Inter $=0$ ) e aqueles que apresentaram pequenos resultados positivos (Inter=1); e $D A_{i t}$ são os accruals discricionários estimado pelo modelo Jones modificado (1995). Foram inseridas variáveis de controle por meio de dummies para cada ano e estado.

Fonte: Elaborada pelos autores.

Como se pode observar, os testes empíricos demonstram que há uma relação positiva significante ( $\mathrm{p}$-value $=0,020)$ entre os accruals discricionários e a probabilidade de municípios apresentarem pequenos superávits, evidenciando a prática de gerenciamento de resultados.

Os resultados vão ao encontro da literatura sobre o tema, apresentando evidências semelhantes às municipalidades de Portugal, onde Ferreira et al. (2013) concluíram que os gestores públicos locais utilizavam práticas oportunistas para apresentar pequenos resultados positivos em suas demonstrações contábeis, em vez de apresentar pequenos resultados negativos. Na subseção 4.5 , foi rodado um modelo adicional, baseado em accruals correntes extremos, com vistas a corroborar os achados.

\subsection{Resultados do Teste de Hipótese H3}

Por fim, foi aplicado o modelo de regressão desenvolvido na sessão 3.2 (Equação 9) para avaliar se a prática de gerenciamento de resultados é maior nos períodos pré-eleitoral e eleitoral, bem como se tal prática é reduzida em ambientes de maior competição política. O resultado encontra-se descrito na Tabela 6. 


\begin{tabular}{|c|c|c|c|c|c|}
\hline \multicolumn{6}{|c|}{ Painel A: Estimativa dos coeficientes obtidos através do modelo de regressão } \\
\hline Variáveis & $\mathrm{N}$ & Coeficientes & Erro padrão & Estatística T & $\mathrm{p}$-value \\
\hline Constante & 10.093 & $-0,004$ & 0,004 & $-0,87$ & 0,384 \\
\hline leleicao & 10.093 & 0,018 & 0,005 & 3,80 & 0,000 \\
\hline Dcomp & 10.093 & $-0,020$ & 0,005 & $-3,86$ & 0,000 \\
\hline \multicolumn{6}{|c|}{ Painel B: Coeficientes de determinação } \\
\hline$R 2$ & 10.093 & 0,002 & & & \\
\hline R2 ajust. & 10.093 & 0,002 & & & \\
\hline
\end{tabular}

A regressão foi calculada utilizando o seguinte modelo: $D A_{i t}=\alpha_{i t}+\beta_{1}\left(\right.$ Ieleição $\left.o_{i t}\right)+\beta_{2}\left(D c o m p_{t+1}\right)+\varepsilon_{i t}$, onde: $D A_{i t}$ são os accruals discricionários estimados pelo modelo Jones modificado (1995); Ieleição é a variável dummy que particiona a amostra entre os períodos pré-eleitoral e eleitoral (2015 e 2016) e os demais períodos (2013 e 2014); Dcomp é a variável dummy que particiona a amostra entre os grupos que apresentam ambiente de alta competitividade (mais de 2 candidatos disputando o cargo) e que apresentam menor competitividade ( 2 ou menos candidatos disputando o cargo) no ano eleitoral, calculada pelo seguinte modelo de Laakso e Taagepera (1979): $\operatorname{Comp}=\frac{1}{\sum_{i=1}^{n} p_{i}^{2}}$, onde $p_{i}^{2}$ é a parte fracionária de votos obtidos durante a eleição para o partido $i$.

Fonte: Elaborada pelos autores.

Como se percebe, há uma relação positiva bastante significativa ( $\mathrm{p}$-value $=0,000)$ entre os períodos pré-eleitoral e eleitoral e os accruals discricionários. Os resultados apresentados corroboram as evidências de comportamento oportunista através de gerenciamento de resultados por parte dos gestores locais em anos pré-eleitorais e eleitorais, com o objetivo de maximizar votos para a reeleição (Buchanan \& Tullok, 1962; Ferreira et al., 2013; Gonçalves et al., 2017).

Quanto à variável de competição política, tal relação também se mostrou bastante significativa (p-value $=0,000)$, porém, inversa aos accruals discricionários observados, demonstrando que ambientes de maior competição política para os governos locais inibem a prática de gerenciamento de resultados por parte dos gestores (Ferreira et al., 2013).

\subsection{Análises Adicionais}

\subsubsection{Modelo de accruals correntes extremos (H2)}

O teste da Hipótese $\mathrm{H} 2$ consiste na avaliação sobre se há relação positiva entre os accruals discricionários $(D A)$ e a probabilidade de apresentação de "pequenos superávits". Em virtude do processo de geração e reversão de accruals, é possível indagar se valores positivos de accruals discricionários correspondem a manipulações para aumentar o superávit ou se são reversões de accruals originados em período anterior, com objetivo de reduzir o superávit.

Como teste adicional, aplicou-se o modelo de Fedyk, Singer e Sougiannis (2020), adaptado para o setor público, que consiste na mensuração dos accruals correntes (TCA).

O passo inicial consiste no cálculo dos accruals correntes (TCA), que correspondem aos accruals totais (TA), adicionando-se accruals de longo prazo, depreciação, amortização e exaustão (deprecia). Portanto, 
TCA = TA + deprecia. Conforme Fedyk et al. (2020), os accruals extremos, positivos ou negativos, corresponderiam àqueles de origem, e não de reversão. Assim, os accruals correntes foram ordenados, e o quartil superior foi considerado como o contendo os accruals correntes positivos de abertura.

Os resultados anteriores apresentados para $\mathrm{H} 2$ indicam relação positiva entre os accruals discricionários $(D A)$ e a probabilidade de o município apresentar um "pequeno superávit". Assim, foi rodado o seguinte modelo Probit para apurar se há relação entre o surgimento de accruals correntes positivos extremos e a probabilidade de apresentação de "pequenos superávits":

$$
\text { Inter }_{i t}=a_{i t}+\beta_{1}\left(P_{o s}{ }_{-} T C A_{i t}\right)+\varepsilon_{i t}
$$

Em que Inter ${ }_{i t}$ representa a variável dummy que divide os municípios em 2 grupos: os que apresentaram "pequenos resultados negativos" (Inter $=0$ ) e os que apresentaram "pequenos resultados positivos" (Inter=1). Pos_TCAit é uma variável dummy que assume 1, se os accruals correntes se encontram no quartil superior, ou 0 , caso contrário.

Os resultados (não tabulados) indicam que há relação positiva e significativa entre o surgimento de accruals positivos extremos e a probabilidade de apresentação de "pequenos superávits", corroborando os achados em relação $\mathrm{H} 2$ aos accruals discricionários. O nível de confiança é de 95\%, quando considerados controles para tamanho do município, competição política e apresentação de déficits em período anterior.

\section{CONCLUSÃO}

O presente estudo teve como objetivo buscar evidências da relação entre a prática de gerenciamento de resultados realizados pelos gestores públicos municipais e o ciclo eleitoral.

Os testes empíricos realizados forneceram evidências para a ocorrência de descontinuidade em torno do resultado nulo (breakeven) e demonstram que os gestores locais tendem a apresentar pequenos resultados positivos, ao invés de negativos, por meio da prática de gerenciamento de resultados. Também foi confirmado o gerenciamento de resultados por parte dos gestores locais brasileiros, notadamente em períodos pré-eleitorais e eleitorais, quando são intensificados gastos com investimentos e podem se intensificar eventuais comportamentos oportunistas dos gestores locais.

De igual modo, restou evidenciado que um ambiente de forte competição política inibe as práticas de gerenciamento, uma vez que o controle social e dos outros partidos amplia o olhar para a gestão atual sobre as escolhas contábeis realizadas (Ferreira et al., 2013).

Os resultados vão ao encontro da literatura que aponta para a existência de motivação para gerenciar resultados na busca pela maximização de votos, visando à reeleição, por meio da tentativa de demonstrar maior eficiência na gestão mediante demonstrativos contábeis (Ferreira et al., 2013). Tal fato acende um sinal de alerta para as entidades de controle do setor público brasileiro, que devem levar em conta os possíveis comportamentos oportunistas por parte dos gestores locais em suas análises dos demonstrativos contábeis.

Este estudo também implica maior atenção dos atores envolvidos no processo de convergência aos padrões internacionais, de modo que as normas contábeis adotadas no Brasil busquem a redução da assimetria informacional e a ampliação da utilidade da informação, evitando que os demonstrativos contábeis sirvam como instrumento de manobra política. 
RAP | Gerenciamento de resultados e ciclo eleitoral em municípios brasileiros

Não obstante, devem-se considerar como limitações do presente trabalho a reduzida quantidade de observações referentes ao exercício de 2013 e o fato de que o processo de convergência às normas internacionais ainda se encontra em transição no âmbito nacional. Além disso, não foram consideradas outras características políticas, como sistema eleitoral de um ou dois turnos, por exemplo.

Como sugestão, novas pesquisas podem ser realizadas nos níveis local e regional no ambiente brasileiro, de modo a acompanhar a ocorrência de práticas oportunistas ligadas às demonstrações financeiras, levando-se em conta o atual processo de convergência e evolução da contabilidade pública nacional. 


\section{REFERÊNCIAS}

Akhmedov, A., \& Zhuravskaya, E. (2004). Opportunistic political cycles: test in a young democracy setting. The Quarterly Journal of Economics, 119(4), 1301-1338.

Baber, W., \& Sen, P. (1986). The political process and the use of debt financing by state governments. Public Choice, 48(3), 201-15.

Barea, T. J. (1994). El Nuevo Plan General de Contabilidad Pública. Revista Española de Financiación y Contabilidad, 29, 335-358.

Buchanan, J., \& Tullok, G. (1962). The calculus of consent. Ann Arbor, Michigan: University of Michigan Press.

Burghstahler, D. C., \& Dichev, I. (1997). Earnings management to avoid earnings decrease and losses. Journal of Accounting and Economics, 24(1), 99-126.

Coelho, C. (2004). Ciclos político-econômicos e o poder local. Braga, Portugal: Universidade do Minho.

Conselho Federal de Contabilidade. (2016). Resolução 2016/NBCTSPEC. Norma Brasileira de Contabilidade - NBC TSP Estrutura Conceitual. Recuperado de https://www2.cfc.org.br/ sisweb/sre/detalhes_sre.aspx?Codigo $=2016 /$ NBCTSPEC\&arquivo $=$ NBCTSPEC. doc

Dechow, P., \& Dichev, I. D. (2002). The quality of accruals and earnings: the role of accrual estimation errors. The Accounting Review, 77, 35-59.

Dechow, P., Ge, W., \& Schrand, C. (2010). Understanding earnings quality: a review of the proxies, their determinants and their consequences. Journal of Accounting and Economics, 50, 344-401.

Dechow, P., Richardson, S. A., \& Tuna, I. (2003). Why are earnings kinky? An explanation of the earnings management explanation. Review of Accounting Studies, 8, 355-384.

Dechow, P., Sloan, R. G., \& Sweeney, A. P. (1995). The Accounting Review, 70, 193-225.

Drazen, A., \& Eslava, M. (2005). Electoral manipulation via voter-friendly spending: theory and evidence. Recuperado de www.tau.ac.il/*drazen/ pbccomp_6292005_ad.pdf

European Federation of Accountants. (2003). The adoption of accrual accounting and budgeting by governments. Recuperado de www. accountancyeurope.eu/publications/the-adoptionof-accrual-accounting-and-budgeting-bygovernments-central-federal-regional-and-local/

Ferreira, A., Carvalho, J., \& Pinho, F. (2013). Earnings management around zero: a motivation to local politician signalling competence. Public Management Review, 15(5), 657-686.

Ferreira, F. R., \& Costa, F. M. (2011). Gerenciamento de resultados e "nova gestão pública": evidências nos departamentos governamentais australianos. In Anais do $5^{\circ}$ Congresso da Associação Nacional dos Programas de Pós-Graduação em Ciências Contábeis, Vitória, ES.

García, F. M. (2014). Developments and challenges in public sector accounting. Journal of Public Budgeting, Accounting \& Financial Management, 26, 345-366.

Gonçalves, L. G., Funchal, B., \& Bezerra, J. E., Filho. (2015). A influência dos ciclos políticos nos investimentos públicos em infraestrutura: um estudo nos estados brasileiros no período de 2003 a 2014 . Revista de Administração Pública, 51(4), 462-481.

Healy, P. M. (1985). The effect of bonus schemes on accounting decisions. Journal of accounting and economics, 7, 85-107.

Healy, P. M., \& Wahlen, J. M. (1999). A review of the earnings management literature and its implications for standard setting. Accounting Horizons, 13(4), 365-383.

Hepwort, N. (2003). Preconditions for successful implementation of accrual accounting in central government. Public Money \& Management, 23, 37-43.

International Monetary Fund. (2014). Government Finance Statistics Manual. Washington, DC: Autor.

International Public Sector Accounting Standards Board. (2016). Handbook of International Public Sector Accounting Pronouncements. New York, NY: Autor.

Jones, J. (1991). Earnings management during import relief investigations. Journal of Accounting Research, 29, 193-228.

Kothari, S. P., Ramana, K., \& Skinner, D. J. (2010). Implications for GAAP from an analysis of positive 
research in accounting. Journal of Accounting and Economics, 50, 246-286.

Laakso, M., Taagepera, R. (1979). "Effective" number of parties - a measure with application to West Europe. Comparative Political Studies, 12(1), 3-27.

Leary, M. R., Kowalski, R. M. (1990). Impression management: a literature review and two-component model. Psychological Bulletin, 107, 34-47.

Lei Complementar n. ${ }^{\circ} 101$, de 4 de maio e 2000. (2000). Estabelece normas de finanças públicas voltadas para a responsabilidade na gestão fiscal e dá outras providências. Recuperado de www.planalto. gov.br/ccivil_03/leis/lcp/lcp101.htm

Lei no 4.320, de 17 de março de 1964. (1964). Estatui normas gerais de direito financeiro para elaboração e controle dos orçamentos e balanços da união, dos estados, dos municípios e do distrito federal. Recuperado de www.planalto.gov.br/ccivil_03/leis/ 14320.htm

Mcnichols, M. F. (2000). Research design issues in earnings management studies. Journal of Accounting and Public Policy, 19, 313-345.

Mcnichols, M. F., \& Wilson, G. P. (1988). Evidence of earnings management from de provisions for bad debts. Journal of Accounting Research, 26, 1-31.

Mota, F. G. L. (2015). Contabilidade aplicada ao setor público: aspectos patrimoniais - identificação, reconhecimento, mensuração e evidenciação. Brasília, DF: Autor.

Nakaguma, M., \& Bender, S. (2006). A emenda da reeleicão e a Lei de Responsabilidade Fiscal: impactos sobre ciclos políticos e performance fiscal dos estados (1986-2002). Economia Aplicada, 10(3), 377-97.

Organização para a Cooperação e o Desenvolvimento Econômico. (2017). Accrual pratices and reform experiences in OECD countries. Paris, France: Autor. Recuperado de http://dx.doi. org/10.1787/9789264270572-en

Pilcher, R., \& Zahn, J. L. W. M. V. (2008). Earnings management and income distribution around a "breakeven or near breakeven surplus" benchmark with the public sector. In Proceedings of $2008 \mathrm{Afaanz}$ Annual Conference, Sydney, Australia.

Portaria $n^{\circ} 184$ de 25 de agosto de 2008. (2008). Dispõe sobre as diretrizes a serem observadas no setor público (pelos entes públicos) quanto aos procedimentos, práticas, elaboração e divulgação das demonstrações contábeis, de forma a tornálos convergentes com as Normas Internacionais de Contabilidade Aplicadas ao Setor Público. Recuperado de www.fazenda.gov.br/acesso-ainformacao/institucional/legislacao/portariasministeriais/2008/portaria184

Portaria $n^{\circ} 548$ de 24 de setembro de 2015. (2015). Dispõe sobre prazos-limite de adoção dos procedimentos contábeis patrimoniais aplicáveis aos entes da Federação, com vistas à consolidação das contas públicas da União, dos estados, do Distrito Federal e dos municípios, sob a mesma base conceitual. Recuperado de www.tesouro.fazenda. gov.br/documents/10180/0/Portaria+STN $+548+20$ 15+PIPCP/73e5e615-ccbe-4050-bfc3-a9356d35daf1

Preussler, A., \& Portugal, M. (2003). Um estudo empírico dos ciclos político-econômicos no Brasil. Análise Econômica, 21(40), 179-205.

Przeworski, A., Stokes, S. C., \& Manin, B. (1999). Democracy, accountability and representation. Cambridge, UK: Cambridge University Press.

Secretaria do Tesouro Nacional. (2017). Manual de contabilidade aplicada ao setor público aplicado à União e aos Estados, Distrito Federal e Municípios (7a ed.). Brasília, DF: Autor.

Silva, V. L. (2012). A nova contabilidade aplicada ao setor público: uma abordagem prática. São Paulo, SP: Atlas.

Wynne, A. (2007, October). Is the move to accrual base accounting a real priority for public sector accounting? Public Fund Digest, 6(1), 1-32.

Zimmerman, J. (1977). The municipal accounting maze: an analysis of political incentives. Journal of Accounting Research, 15, 107-44. 


\section{Fábio Moraes da Costa}

https://orcid.org/0000-0002-2098-4006

Doutor em Ciências Contábeis pela Universidade de São Paulo (USP); Professor associado da FUCAPE Business School. E-mail: fabio@fucape.br

\section{Fernando Henrique Ferreira Carneiro Leão}

https://orcid.org/0000-0001-8636-8129

Mestre em Ciências Contábeis pela FUCAPE Business School, Vitória - ES; Auditor de Controle Externo do Tribunal de Contas do Estado do Rio de Janeiro (TCE-RJ); Professor dos cursos de pós-graduação da Escola de Contas e Gestão do TCE-RJ. E-mail: fernandoleao@gmail.com 